\title{
FRAME FOR OPERATORS IN FINITE DIMENSIONAL HILBERT SPACE
}

\author{
VAHID REZA MORSHEDI, MOHAMMAD JANFADA AND RAJABALI KAMYABI GOL
}

\begin{abstract}
In this paper, we study frames for operators ( $K$-frames) in finite dimensional Hilbert spaces and express the dual of $K$-frames. Some properties of $K$-dual frames are investigated. Furthermore, the notion of their oblique $K$-dual and some properties are presented.
\end{abstract}

\section{Introduction}

Frames were first introduced by Duffin and Schaeffer in the study of nonharmonic Fourier series in 1952, [8], and were widely studied from 1986 since the great work by Daubechies et al, [7]. Now, frames play an important role not only in the theoretic but also in many kinds of applications, for example, signal processing [10], filter bank theory [3] and many other fields $[9,13,15]$.

The notion of $K$-frames was considered for the first time in [11], in connection with atomic decompositions for operators in Hilbert spaces. Basic properties and examples of $K$-frames are given in [11] and [12].

Let $K \in B(\mathscr{H})$, the space of all bounded linear operators on a Hilbert space $\mathscr{H}$. A sequence $\left\{\varphi_{j}\right\}_{j \in \downarrow}$ is said to be a $K$-frame for $\mathscr{H}$ if there exist constants $A, B>0$ such that

$$
A\left\|K^{*} x\right\|^{2} \leq \sum_{j \in \rrbracket}\left|\left\langle x, \varphi_{j}\right\rangle\right|^{2} \leq B\|x\|^{2}, \quad(x \in \mathscr{H}) .
$$

We call $A, B$ the lower and the upper $K$-frame bounds for $\left\{\varphi_{j}\right\}_{j \in \sqrt{ }}$, respectively. If $K=I_{\mathscr{H}}$, then $\left\{\varphi_{j}\right\}_{j \in \downarrow}$ is the ordinary frame. If only the right inequality holds, then $\left\{\varphi_{j}\right\}_{j \in \downarrow}$ is called a Bessel sequence. Suppose that $\Phi=\left\{\varphi_{j}\right\}_{j \in \downarrow}$ is a $K$-frame for $\mathscr{H}$. The operator $T_{\Phi}: \mathscr{H} \rightarrow \ell^{2}(\mathbb{V})$ defined by $T_{\Phi}(x)=\left\{\left\langle x, \varphi_{j}\right\rangle\right\}_{j \in \downarrow}$ is called the analysis operator. $T_{\Phi}$ is bounded and $T_{\Phi}^{*}: \ell^{2}(\mathbb{J}) \rightarrow$ $\mathscr{H}$ is given by $T_{\Phi}^{*}\left(\left\{c_{j}\right\}_{j \in \rrbracket}\right)=\sum_{j \in \rrbracket} c_{j} \varphi_{j} . T_{\Phi}^{*}$ is called the pre-frame or synthesis operator. The

Received December 20, 2016, accepted November 17, 2017. 2010 Mathematics Subject Classification. 42C15, $46 \mathrm{~B} 15$.

Key words and phrases. $K$-frame, $K$-dual, oblique $K$-dual. Corresponding author: Mohammad Janfada. 
operator $S_{\Phi}: \not{H} \rightarrow \mathscr{H}$ defined by $S_{\Phi}(x)=T_{\Phi}^{*} T_{\Phi}(x)=\sum_{j \in \downarrow}\left\langle x, \varphi_{j}\right\rangle \varphi_{j}$ is called the frame operator of $\Phi$. Note that, frame operator of a $K$-frame is not invertible on $\mathscr{H}$ in general, but it is invertible on the subspace $R(K) \subset \mathscr{H}$, that $R(K)$ is the range of $K$, and for all $x \in S_{\Phi}(R(K))$ we have

$$
B^{-1}\|x\| \leq\left\|S_{\Phi}^{-1} x\right\| \leq A^{-1}\left\|K^{\dagger}\right\|^{2}\|x\|
$$

where $K^{\dagger}$ is the pseudo-inverse of $K$. If $K$ is invertible then $S_{\Phi}$ is invertible. Also, in this case, we can see that any $K$-frame for $\mathscr{H}^{N}$ is a frame for $\mathscr{H}^{N}$. Since we can write

$$
\frac{A}{\left\|K^{-1}\right\|^{2}}\|x\|^{2} \leq A\left\|K^{*} x\right\|^{2} \leq \sum_{j=1}^{M}\left|\left\langle x, \varphi_{j}\right\rangle\right|^{2}
$$

and for all $x \in \mathscr{H}^{N}$ we have

$$
A\|x\|^{2}=A\left\|K^{*}\left(K^{*}\right)^{-1} x\right\|^{2} \leq \sum_{j=1}^{M}\left|\left\langle\left(K^{*}\right)^{-1} x, \varphi_{j}\right\rangle\right|^{2}=\sum_{j=1}^{M}\left|\left\langle x, K^{-1} \varphi_{j}\right\rangle\right|^{2} .
$$

Hence the sequence $\left\{K^{-1} \varphi_{j}\right\}_{j=1}^{M}$ is a frame.

Given a positive integer $N$. Throughout this paper, we suppose that $\mathscr{H}^{N}$ is a real or complex $N$-dimensional Hilbert space. By $\langle\cdot, \cdot\rangle$ and $\|$.$\| we denote the inner product on \mathscr{H}^{N}$ and its corresponding norm, respectively. $I_{H}$ is the identity operator on $\mathscr{H}^{N}$. For two Hilbert spaces $\mathscr{H}_{1}$ and $H_{2}$ we denote by $B\left(\mathscr{H}_{1}, \mathscr{H}_{2}\right)$ the collection of all bounded linear operators between $\mathscr{H}_{1}$ and $\mathscr{H}_{2}$, and we abbreviate $B(\mathscr{H}, \mathscr{H})$ by $B(\mathscr{H})$. In particular, $B\left(\mathscr{C}^{N}\right)=\mathscr{M}_{N \times N}(\mathbb{C})$. Denote by $P_{W}$ the orthogonal projection of $\mathscr{H}$ onto a closed subspace $W \subseteq \mathscr{H}$.

Finite frames and its properties were proposed by several researchers. In particular, the theory of finite frames in $\mathscr{H}^{N}$ was developed by P. G. Casazza et al [4]. Also, the concept of oblique dual frames and their properties in finite dimensional Hilbert space were presented by X. C. Xiao, Y. C. Zhu and X. M. Zeng [18].

The paper is organized in the following manner. In Section 2, we study the notion of a finite $K$-frames and prove some properties in finite dimensional Hilbert space. In particular, we give a simple way to construct new $K$-frames from given ones. Also, we extend Theorem 1.1 in [10] to the setting of $K$-dual frame pairs. In Section 3, we introduce the concept of $K$ dual of $K$-frames in $\mathscr{H}^{N}$ and its properties are discussed. Also, in the last part of Section 3, the oblique $K$-dual is investigated.

\section{Finite $K$-frame}

Frames in finite dimensional spaces, i.e., finite frames, are a very important class of frames due to their significant relevance in applications. In this section, we present $K$-frame 
theory in finite-dimensional Hilbert spaces. Let $K \in B\left(\mathscr{H}^{N}\right)$ and $\Phi=\left\{\varphi_{j}\right\}_{j=1}^{M}$ be a family of vectors in $\mathscr{H}^{N}$. If $A\left\|K^{*} x\right\|^{2}=\sum_{j=1}^{M}\left|\left\langle x, \varphi_{j}\right\rangle\right|^{2}$, then $\Phi$ is called an $A$-tight $K$-frame and if $\left\|K^{*} x\right\|^{2}=\sum_{j=1}^{M}\left|\left\langle x, \varphi_{j}\right\rangle\right|^{2}$, then $\Phi$ is called a tight $K$-frame or Parseval $K$-frame. We can see that, $\Phi$ is an $A$-tight $K$-frame if and only if $S_{\Phi}=A K K^{*}$. If $\left\|\varphi_{j}\right\|=1$ for all $j=1,2, \ldots, M$, this is an unit norm $K$-frame. Also, if a $K$-frame $\Phi$ is independent in $\mathscr{H}^{N}$ then it is call minimal $K$-frame.

Note that, if $K \in B\left(\mathscr{H}^{N}\right)$ and $\Phi=\left\{\varphi_{j}\right\}_{j=1}^{M}$ is a frame for $H^{N}$, then $\Phi$ is a $K$-frame. Since for any $K \neq 0$ we can write

$$
\frac{A}{\|K\|^{2}}\left\|K^{*} x\right\|^{2} \leq A\|x\|^{2} \leq \sum_{j=1}^{M}\left|\left\langle x, \varphi_{j}\right\rangle\right|^{2},\left(x \in \mathscr{P}^{N}\right) .
$$

\section{Remark 2.1.}

(i) If $\operatorname{span}\left\{\varphi_{j}: j=1,2, \ldots, M\right\}=R(K)$, then $\left\{\varphi_{j}\right\}_{j=1}^{M}$ is a frame for $R(K)$. Thus $\left\{\varphi_{j}\right\}_{j=1}^{M}$ is a $K$-frame for $R(K)$.

(ii) Any $K$-frame is not necessary a frame, in general. For example, let $\mathscr{H}^{3}=\mathbb{R}^{3}$ and $K=P_{\mathbb{R}^{2}}$. Then $\left\{e_{1}, e_{2}\right\}$ is a $K$-frame which is not a frame since

$$
\operatorname{span}\left\{e_{1}, e_{2}\right\} \neq \mathbb{R}^{3}
$$

Note that, if $\Phi$ is a $K$-frame for $\mathscr{H}^{N}$, then by Proposition 3.1 in [17], there exists a sequence $\Psi=\left\{\psi_{j}\right\}_{j=1}^{M} \subseteq \mathscr{H}^{N}$ such that

$$
K x=\sum_{j=1}^{M}\left\langle x, \psi_{j}\right\rangle \varphi_{j},\left(x \in \mathscr{H}^{N}\right)
$$

and this means that $R(K) \subseteq \operatorname{span}\left\{\varphi_{j}: j=1,2, \ldots, M\right\}$. Furthermore, we have the following proposition.

Proposition 2.2 ([16]). A sequence $\Phi=\left\{\varphi_{j}\right\}_{j=1}^{M}$ is a $K$-frame for $\mathscr{H}^{N}$ if and only if $R(K) \subseteq$ $\operatorname{span}\left\{\varphi_{j}: j=1,2, \ldots, M\right\}$.

Now, for an arbitrary $K$-frame, we obtain the optimal lower and upper $K$-frame bounds by eigenvalues of its frame operator.

\section{Proposition 2.3.}

(i) Let $0 \neq K \in B\left(\mathscr{H}^{N}\right)$. Let $\Phi=\left\{\varphi_{j}\right\}_{j=1}^{M}$ be a $K$-frame for $R(K)$ with $K$-frame operator $S_{\Phi}$ with eigenvalues $\lambda_{1} \geq \lambda_{2} \geq \cdots \geq \lambda_{N}>0$. Then $\lambda_{1}$ is the optimal upper $K$-frame bound and if $\lambda_{N} \neq 0$ then $\frac{\lambda_{N}}{\|K\|^{2}}$ is the optimal lower $K$-frame bound. 
(ii) Suppose that $\Phi=\left\{\varphi_{j}\right\}_{j=1}^{M}$ is a $K$-frame for $R(K)$ and $\left\{\lambda_{j}\right\}_{j=1}^{N}$ denotes the eigenvalues for $S_{\Phi}$ and each eigenvalue appears in the list corresponding to its algebraic multiplicity. Then

$$
\sum_{j=1}^{N} \lambda_{j}=\sum_{j=1}^{M}\left\|\varphi_{j}\right\|^{2}
$$

Proof. For the proof of (i), suppose that $\left\{e_{j}\right\}_{j=1}^{N}$ is an orthonormal eigen basis of the frame operator $S_{\Phi}$ with associated eigenvalues $\left\{\lambda_{j}\right\}_{j=1}^{N}$ given in decreasing order. Hence we can write $x=\sum_{j=1}^{N}\left\langle x, e_{j}\right\rangle e_{j}$, for all $x \in \mathscr{H}^{N}$. Also, we have

$$
S_{\Phi} x=\sum_{j=1}^{N}\left\langle x, e_{j}\right\rangle S_{\Phi} e_{j}=\sum_{j=1}^{N} \lambda_{j}\left\langle x, e_{j}\right\rangle e_{j},
$$

and thus

$$
\begin{aligned}
\sum_{j=1}^{M}\left|\left\langle x, \varphi_{j}\right\rangle\right|^{2}= & \left\langle S_{\Phi} x, x\right\rangle=\sum_{j=1}^{N} \lambda_{j}\left|\left\langle x, e_{j}\right\rangle\right|^{2} \\
& \leq \lambda_{1} \sum_{j=1}^{N}\left|\left\langle x, e_{j}\right\rangle\right|^{2}=\lambda_{1}\|x\|^{2} .
\end{aligned}
$$

For the lower bound we have

$$
\begin{aligned}
\frac{\lambda_{N}}{\|K\|^{2}}\left\|K^{*} x\right\|^{2} & \leq \lambda_{N}\|x\|^{2}=\lambda_{N} \sum_{j=1}^{M}\left|\left\langle x, e_{j}\right\rangle\right|^{2} \\
& \leq \sum_{j=1}^{N} \lambda_{j}\left|\left\langle x, e_{j}\right\rangle\right|^{2}=\left\langle S_{\Phi} x, x\right\rangle .
\end{aligned}
$$

The proof of secondly part is similar to the proof of Theorem 1.1.12 [6].

Now, we introduce a constructive method to extend a given frame to a tight $K$-frame.

Theorem 2.4. Let $K \in B\left(\mathscr{H}^{N}\right)$. Let $\Phi=\left\{\varphi_{j}\right\}_{j=1}^{M}$ be a frame for $\mathscr{H}^{N}$. Assume that the frame operator $S_{\Phi}$ has the eigenvalues $\left\{\lambda_{j}\right\}_{j=1}^{N}$, ordered as $\lambda_{1} \geq \lambda_{2} \geq \cdots \geq \lambda_{N}>0$. Let $\left\{e_{j}\right\}_{j=1}^{N}$ be a corresponding eigenbasis. Then the collection $\left\{K \varphi_{j}\right\}_{j=1}^{M} \cup\left\{\sqrt{\lambda_{1}-\lambda_{j}} K e_{j}\right\}_{j=2}^{N}$ is a $\lambda_{1}$-tight Kframe for $\mathscr{H}^{N}$.

Proof. Set $\left\{\psi_{j}\right\}_{j=2}^{N}=\left\{\sqrt{\lambda_{1}-\lambda_{j}} K e_{j}\right\}_{j=2}^{N}$. Suppose that $\tilde{S}$ is the frame operator for $\left\{K \varphi_{j}\right\}_{j=1}^{M} \cup$ $\left\{\psi_{j}\right\}_{j=2}^{N}$. Now, for any $x \in \mathscr{H}^{N}$ we can write

$$
\begin{aligned}
\tilde{S} x & =\sum_{j=1}^{M}\left\langle x, K \varphi_{j}\right\rangle K \varphi_{j}+\sum_{j=2}^{M}\left\langle x, \psi_{j}\right\rangle \psi_{j} \\
& =K \sum_{j=1}^{M}\left\langle K^{*} x, \varphi_{j}\right\rangle \varphi_{j}+\sum_{j=2}^{M}\left\langle x, \psi_{j}\right\rangle \psi_{j}
\end{aligned}
$$




$$
\begin{aligned}
& =K \sum_{j=1}^{N} \lambda_{j}\left\langle K^{*} x, e_{j}\right\rangle e_{j}+K \sum_{j=2}^{N} \sqrt{\lambda_{1}-\lambda_{j}}\left\langle K^{*} x, e_{j}\right\rangle \sqrt{\lambda_{1}-\lambda_{j}} e_{j} \\
& =K \sum_{j=1}^{N} \lambda_{j}\left\langle K^{*} x, e_{j}\right\rangle e_{j}+K \sum_{j=2}^{N}\left(\lambda_{1}-\lambda_{j}\right)\left\langle K^{*} x, e_{j}\right\rangle e_{j} \\
& =\lambda_{1} K K^{*} x .
\end{aligned}
$$

Therefore $\left\{K \varphi_{j}\right\}_{j=1}^{M} \cup\left\{\sqrt{\lambda_{1}-\lambda_{j}} K e_{j}\right\}_{j=2}^{N}$ is a $\lambda_{1}$-tight $K$-frame for $\mathscr{H}^{N}$.

Example 2.5. Let $K \in B\left(\mathscr{H}^{6}\right)$. Consider the frame $\Phi=\left\{\varphi_{j}\right\}_{j=1}^{5}:=\left\{3 e_{1}\right\} \cup\left\{e_{j}\right\}_{j=2}^{5}$ for the subspace $W=\operatorname{span}\left\{e_{j}, j=1,2, \ldots, 5\right\}$ of $\mathscr{P}^{6}=\mathbb{C}^{6}$ where $\left\{e_{j}\right\}_{j=1}^{6}$ is the orthonormal basis for $\mathscr{H}^{6}$. We have

$$
S_{\Phi} x=\sum_{j=1}^{5} \lambda_{j}\left\langle x, e_{j}\right\rangle e_{j}=\sum_{j=1}^{5}\left\langle x, \varphi_{j}\right\rangle \varphi_{j},(x \in W) .
$$

Thus we can see that the eigenvalues for the frame operator $S_{\Phi}$ are $\lambda_{1}=9, \lambda_{j}=1, j=$ $2, \ldots, 5$. Hence by Theorem 2.1 there exist 4 vectors $\left\{\psi_{j}\right\}_{j=1}^{4}=\left\{\sqrt{9-\lambda_{j}} K e_{j}\right\}_{j=2}^{5}$ such that $\left\{K \varphi_{j}\right\}_{j=1}^{5} \cup\left\{\sqrt{9-\lambda_{j}} K e_{j}\right\}_{j=2}^{5}$ is a 9-tight $K$-frame for $W$.

In the following proposition, we express two inequality of $A$-tight $K$-frames.

\section{Proposition 2.6.}

(i) If $\Phi=\left\{\varphi_{j}\right\}_{j=1}^{M}$ is an A-tight K-frame for $\mathscr{H}^{N}$, then

$$
\max _{j=1,2, \ldots, M}\left\|\varphi_{j}\right\|^{2} \leq A\|K\|^{2} .
$$

(ii) If $\Phi=\left\{\varphi_{j}\right\}_{j=1}^{M}$ is an unit norm A-tight $K$-frame for $\mathscr{H}^{N}$, then

$$
A\|K\|^{2} N \geq M
$$

\section{Proof.}

(i) Note that for any $j=1, \ldots, M$, we have

$$
\left\|\varphi_{j}\right\|^{4}=\left|\left\langle\varphi_{j}, \varphi_{j}\right\rangle\right|^{2} \leq \sum_{i=1}^{M}\left|\left\langle\varphi_{j}, \varphi_{i}\right\rangle\right|^{2}=A\left\|K^{*} \varphi_{j}\right\|^{2} \leq A\left\|K^{*}\right\|^{2}\left\|\varphi_{j}\right\|^{2} .
$$

Thus, $\max _{j=1,2, \ldots, M}\left\|\varphi_{j}\right\|^{2} \leq A\|K\|^{2}$.

(ii) Let $\left\{e_{i}\right\}_{i=1}^{N}$ be an orthonormal basis for $\mathscr{H}^{N}$. Then we can write

$$
\begin{aligned}
M & =\sum_{j=1}^{M}\left\|\varphi_{j}\right\|^{2}=\sum_{j=1}^{M} \sum_{i=1}^{N}\left|\left\langle e_{i}, \varphi_{j}\right\rangle\right|^{2}=\sum_{i=1}^{N} \sum_{j=1}^{M}\left|\left\langle e_{i}, \varphi_{j}\right\rangle\right|^{2} \\
& =\sum_{i=1}^{N} A\left\|K^{*} e_{i}\right\|^{2} \leq A\|K\|^{2} \sum_{i=1}^{N}\left\|e_{i}\right\|^{2}=A\|K\|^{2} N .
\end{aligned}
$$


Remark 2.7. In the frame setting, if $\Phi=\left\{\varphi_{j}\right\}_{j=1}^{M}$ is a finite sequence which is a frame for $\mathscr{H}$, then $\mathscr{H}$ is finite-dimensional [2]. But in the $K$-frame setting, it is not true. For example, we define $K: \ell^{2} \longrightarrow \ell^{2}$ with $K x=\sum_{j=1}^{M}\left\langle x, e_{j}\right\rangle e_{j}$. Clearly $\left\{K e_{j}\right\}_{j=1}^{\infty}=\left\{e_{j}\right\}_{j=1}^{M}$ is a $K$-frame but $\ell^{2}$ is not finite-dimensional.

If we have information on the lower $K$-frame bound of an unit norm $K$-frame, we can provide a criterion for how many elements we can remove so that the rest of the elements forms a $K$-frame.

Proposition 2.8. Suppose that $0 \neq K \in B\left(\mathscr{H}^{N}\right)$ and $\Phi=\left\{\varphi_{j}\right\}_{j=1}^{M}$ is an unit norm $K$-frame for $\mathscr{H}^{N}$ with the lower $K$-frame bound $A$. Then for any index set $I \subseteq\{1, \ldots, M\}$ such that $|I|$ $\left\|\left.K^{\dagger}\right|_{R(K)}\right\|^{2}<A$, the family $\left\{\varphi_{j}\right\}_{j \notin I}$ is a $K$-frame for $R(K)$.

Proof. Let $\Phi=\left\{\varphi_{j}\right\}_{j=1}^{N}$ be an unit norm $K$-frame for $\mathscr{H}^{N}$, then we can write

$$
\sum_{j \in I}\left|\left\langle x, \varphi_{j}\right\rangle\right|^{2} \leq \sum_{j \in I}\left\|\varphi_{j}\right\|^{2}\|x\|^{2}=|I|\|x\|^{2},(x \in R(K)) .
$$

Now, since for any $x \in R(K)$

$$
\frac{A}{\left\|\left.K^{\dagger}\right|_{R(K)}\right\|^{2}}\|x\|^{2} \leq A\left\|K^{*} x\right\|^{2} \leq \sum_{j \in I}\left|\left\langle x, \varphi_{j}\right\rangle\right|^{2}+\sum_{j \notin I}\left|\left\langle x, \varphi_{j}\right\rangle\right|^{2} .
$$

So we have

$$
\left(\frac{A}{\left\|\left.K^{\dagger}\right|_{R(K)}\right\|^{2}}-|I|\right)\|x\|^{2} \leq \sum_{j \notin I}\left|\left\langle x, \varphi_{j}\right\rangle\right|^{2},(x \in R(K)) .
$$

Then $\Phi$ is a frame and hence is a $K$-frame for $R(K)$.

In the last part of this section, we study conditions under which a linear combination of two $K$-frames is $K$-frame too.

Definition 2.9. Let $K \in B\left(\mathscr{H}^{N}\right)$ and $\Phi=\left\{\varphi_{j}\right\}_{j=1}^{M}$ and $\Psi=\left\{\psi_{j}\right\}_{j=1}^{M}$ be $K$-frames for $\mathscr{H}^{N}$. $\Phi$ and $\Psi$ are called strongly disjoint if $R\left(T_{\Phi}\right) \perp R\left(T_{\Psi}\right)$, where $T_{\Phi}$ and $T_{\Psi}$ are the analysis operators of the sequences $\Phi$ and $\Psi$, respectively.

Theorem 2.10. Suppose that $K \in B\left(\mathscr{H}^{N}\right)$ and $\Phi=\left\{\varphi_{j}\right\}_{j=1}^{M}$ and $\Psi=\left\{\psi_{j}\right\}_{j=1}^{M}$ are strongly disjoint tight $K$-frames for $\mathscr{H}^{N}$. Also, assume that $A, B \in B\left(\mathscr{H}^{N}\right)$ are operators such that $A K K^{*} A^{*}+$ $B K K^{*} B^{*}=I_{N \times N}$, then $\{A \Phi+B \Psi\}$ is a $K$-frame for $\mathscr{H}^{N}$. In particular, if $K K^{*}=\frac{1}{2\left(|\alpha|^{2}+|\beta|^{2}\right)} I_{N \times N}$, then $\{\alpha \Phi+\beta \Psi\}$ is a $K$-frame for $\mathscr{H}^{N}$.

Proof. For any $x \in \mathscr{H}^{N}$

$$
\sum_{j=1}^{M}\left|\left\langle x, A \varphi_{j}+B \psi_{j}\right\rangle\right|^{2}=\left\|\left\{\left\langle x, A \varphi_{j}+B \psi_{j}\right\rangle\right\}_{j=1}^{M}\right\|^{2}
$$




$$
\begin{aligned}
& =\left\|\left\{\left\langle A^{*} x, \varphi_{j}\right\rangle+\left\langle B^{*} x, \psi_{j}\right\rangle\right\}_{j=1}^{M}\right\|^{2} \\
& =\left\|\left\{\left\langle A^{*} x, \varphi_{j}\right\rangle\right\}_{j=1}^{M}\right\|^{2}+\left\|\left\{\left\langle B^{*} x, \psi_{j}\right\rangle\right\}_{j=1}^{M}\right\|^{2} \\
& \left.\left.+\left\langle\left\{A^{*} x, \varphi_{j}\right\rangle\right\}_{j=1}^{M},\left\{B^{*} x, \psi_{j}\right\rangle\right\}_{j=1}^{M}\right\rangle_{\mathcal{X}^{N}} \\
& =\sum_{j=1}^{M}\left|\left\langle A^{*} x, \varphi_{j}\right\rangle\right|^{2}+\sum_{j=1}^{M}\left|\left\langle B^{*} x, \psi_{j}\right\rangle\right|^{2} \\
& =\left\|K^{*} A^{*} x\right\|^{2}+\left\|K^{*} B^{*} x\right\|^{2}=\|x\|^{2} .
\end{aligned}
$$

Therefore $\left\{A \varphi_{j}+B \psi_{j}\right\}_{j=1}^{M}$ is a tight frame for $\mathscr{H}^{N}$ and so is a $K$-frame for $\mathscr{H}^{N}$.

Corollary 2.11. Assume that $\left(\Phi_{1}, \Phi_{2}, \ldots, \Phi_{k}\right)$ is strongly disjoint $k$-tuple of tight $K$-frames on $\mathscr{H}^{N}$ and $A_{i} \in B\left(\mathscr{H}^{N}\right)$ such that $\sum_{i=1}^{k} A_{i} K K^{*} A_{i}^{*}=I_{N \times N}$. Then $\left\{\sum_{i=1}^{k} A_{i} \Phi_{i}\right\}$ is a $K$-frame for $\mathscr{H}^{N}$.

Proposition 2.12. With assumption of Theorem 2.2, if $A K K^{*} A^{*}+B K K^{*} B^{*}=K K^{*} I_{N \times N}$, then $\{A \Phi+B \Psi\}$ is a tight $K$-frame for $\mathscr{H}^{N}$.

Remark 2.13. Theorem 2.2, Corollary 2.1 and Proposition 2.5 actually hold in infinite-dimensional Hilbert spaces.

\section{Dual of $K$-frame}

Dual frames are important to reconstruct of vectors (or signals) in terms of the frame elements. In the other words, two frames $\Phi=\left\{\varphi_{j}\right\}_{j \in \rrbracket}$ and $\Psi=\left\{\psi_{j}\right\}_{j \in \rrbracket}$ are dual frames for $\mathscr{H}$ if for all $x \in \mathscr{H}$,

$$
x=\sum_{j \in \rrbracket}\left\langle x, \psi_{j}\right\rangle \varphi_{j}=\sum_{j \in \rrbracket}\left\langle x, \varphi_{j}\right\rangle \psi_{j} .
$$

Also, if $\left\{\varphi_{j}\right\}_{j \in \rrbracket}$ is a $K$-frame, a Bessel sequence $\Psi=\left\{\psi_{j}\right\}_{j \in \rrbracket}$ is called a $K$-dual of $\left\{\varphi_{j}\right\}_{j \in \rrbracket}$ (see [1]) if

$$
K x=\sum_{j \in \rrbracket}\left\langle x, \psi_{j}\right\rangle \varphi_{j}, \quad(x \in \mathscr{H}) .
$$

We can see that, for every $K$-frame of $\mathscr{C}$ there exists at least a Bessel sequence $\left\{\psi_{j}\right\}_{j \in \rrbracket}$ which satisfies in $K$-dual equality and the sequences $\left\{\varphi_{j}\right\}_{j \in \rrbracket}$ and $\left\{\psi_{j}\right\}_{j \in \rrbracket}$ in (3.2) are not interchangeable in general [17]. Now, we study this notion in finite dimensional Hilbert spaces. Definition 3.1. If $\Phi=\left\{\varphi_{j}\right\}_{j=1}^{M}$ is a $K$-frame for $\mathscr{H}^{N}$, a sequence $\Psi=\left\{\psi_{j}\right\}_{j=1}^{M}$ is called a $K$-dual frame for $\Phi$ if

$$
K x=\sum_{j=1}^{M}\left\langle x, \psi_{j}\right\rangle \varphi_{j},\left(x \in \mathscr{H}^{N}\right) .
$$

The systems $\Phi=\left\{\varphi_{j}\right\}_{j=1}^{M}$ and $\Psi=\left\{\psi_{j}\right\}_{j=1}^{M}$ are referred to as a $K$-dual frame pair. 
If $T_{\Phi}^{*}$ and $T_{\Psi}^{*}$ are the $N \times M$ matrices whose $j$-th columns are $\Phi=\left\{\varphi_{j}\right\}_{j=1}^{M}$ and $\Psi=\left\{\psi_{j}\right\}_{j=1}^{M}$, respectively, then (3.3) is equivalent to $K=T_{\Phi}^{*} T_{\Psi}$.

Note that $\Phi=\left\{\varphi_{j}\right\}_{j=1}^{M}$ and $\Psi=\left\{\psi_{j}\right\}_{j=1}^{M}$ are not interchangeable in general. Indeed, $\Phi=$ $\left\{\varphi_{j}\right\}_{j=1}^{M}$ and $\Psi=\left\{\psi_{j}\right\}_{j=1}^{M}$ in (3.3) are interchangeable if and only if $K$ is self adjoint. Also, always there is at least a $K$-dual of any arbitrary $K$-frame.

Example 3.2. Consider the standard orthonormal basis $\Psi=\left\{\psi_{j}\right\}_{j=1}^{3}=\left\{e_{j}\right\}_{j=1}^{3}$ of Hilbert space $\mathbb{C}^{3}$. Define $K \in B\left(\mathbb{C}^{3}\right)$ as follows $K e_{1}=e_{1}, K e_{2}=e_{1}, K e_{3}=e_{2}$. Set $\Phi=\left\{\phi_{j}\right\}_{j=1}^{3}=\left\{K e_{j}\right\}_{j=1}^{3}$, that is, $\Phi=\left\{\phi_{j}\right\}_{j=1}^{3}$ is a $K$-frame. Since $x=\sum_{j=1}^{3}\left\langle x, e_{j}\right\rangle e_{j}, x \in \mathbb{C}^{3}$, then $K x=\sum_{j=1}^{3}\left\langle x, \psi_{j}\right\rangle \varphi_{j}$. Hence $\Psi$ is a $K$-dual of $\Phi$ which is not interchangeable. The frame operator of $\Phi$ is $S=\left[\begin{array}{lll}2 & 0 & 0 \\ 0 & 1 & 0 \\ 0 & 0 & 0\end{array}\right]$.

In the following proposition, trace formula for a tight $K$-frames is stated that is associated to its $K$-dual.

Proposition 3.3. Let $\Phi=\left\{\varphi_{j}\right\}_{j=1}^{M}$ be a tight $K$-frame for $\mathscr{H}^{N}$. Then

$$
\operatorname{Tr}(K)=\sum_{j=1}^{M}\left\langle\varphi_{j}, \psi_{j}\right\rangle,
$$

where $\Psi=\left\{\psi_{j}\right\}_{j=1}^{M}$ is a $K$-dual of $\Phi=\left\{\varphi_{j}\right\}_{j=1}^{M}$.

Proof. We know that if $\left\{e_{i}\right\}_{i=1}^{N}$ is an arbitrary orthonormal basis for $\mathscr{H}^{N}$, then by definition, $\operatorname{Tr}(K)=\sum_{i=1}^{N}\left\langle K e_{i}, e_{i}\right\rangle$. Now, we can write

$$
\begin{aligned}
\operatorname{Tr}(K) & =\sum_{i=1}^{N}\left\langle K e_{i}, e_{i}\right\rangle=\sum_{i=1}^{N}\left\langle\sum_{j=1}^{M}\left\langle e_{i}, \psi_{j}\right\rangle \varphi_{j}, e_{i}\right\rangle=\sum_{j=1}^{M} \sum_{i=1}^{N}\left\langle e_{i}, \psi_{j}\right\rangle\left\langle\varphi_{j}, e_{i}\right\rangle \\
& =\sum_{j=1}^{M} \sum_{i=1}^{N}\left\langle\varphi_{j}, e_{i}\right\rangle\left\langle e_{i}, \psi_{j}\right\rangle=\sum_{j=1}^{M}\left\langle\sum_{i=1}^{N}\left\langle\varphi_{j}, e_{i}\right\rangle e_{i}, \psi_{j}\right\rangle=\sum_{j=1}^{M}\left\langle\varphi_{j}, \psi_{j}\right\rangle .
\end{aligned}
$$

In the following theorem, we characterize the scalar sequences $v=\left\{v_{j}\right\}_{j=1}^{M}$ for which there exists a $K$-dual pair of frames $\left\{\varphi_{j}\right\}_{j=1}^{M}$ and $\left\{\psi_{j}\right\}_{j=1}^{M}$ such that $v_{j}=\left\langle\varphi_{j}, \psi_{j}\right\rangle$ for all $j=$ $1,2, M$.

Theorem 3.4. Let $K \in B\left(\mathscr{H}^{N}\right)$ and $v=\left\{v_{j}\right\}_{j=1}^{M} \subset \mathbb{C}$ with $M>\operatorname{dim}(R(K))=\operatorname{rank}(K)$ be given. Suppose that there exist $K$-dual frame pairs $\left\{\varphi_{j}\right\}_{j=1}^{M}$ and $\left\{\psi_{j}\right\}_{j=1}^{M}$ for $\mathscr{H}^{N}$ such that $v_{j}=\left\langle\varphi_{j}, \psi_{j}\right\rangle$ for all $j=1,2, M$. Then there exists a tight $K^{*}$-frame $\left\{\theta_{j}\right\}_{j=1}^{M}$ and a corresponding dual frame $\Gamma=\left\{\gamma_{j}\right\}_{j=1}^{M}$ for $\mathscr{H}^{N}$ such that $v_{j}=\left\langle\theta_{j}, \gamma_{j}\right\rangle$ for all $j=1,2, M$. Furthermore $\operatorname{Tr}(K)=\sum_{j=1}^{M} v_{j}$. 
Proof. Fix $v=\left\{v_{j}\right\}_{j=1}^{M} \subset \mathbb{C}$ such that there exists a $K$-dual frame pairs $\left\{\varphi_{j}\right\}_{j=1}^{M}$ and $\left\{\psi_{j}\right\}_{j=1}^{M}$ such that satisfying $v_{j}=\left\langle\varphi_{j}, \psi_{j}\right\rangle$ for all $j=1,2, M$. Hence we can write $T_{\Phi}^{*} T_{\Psi}=K$ and $v=$ $\operatorname{diag}\left(T_{\Phi}^{*} T_{\Psi}\right)$, where $\operatorname{diag}(\cdot)$ denotes the column vector of entries on the main diagonal of a matrix. Set $\theta_{j}=K^{*} S_{\Phi}^{-\frac{1}{2}} P_{S_{\Phi}^{\frac{1}{2}}(R(K))} \varphi_{j}$ and $\gamma_{j}=S_{\Phi}^{\frac{1}{2}}\left(\left.K^{\dagger}\right|_{R(K)}\right)^{*} \psi_{j}$. Thus $\Theta=\left\{\theta_{j}\right\}_{j=1}^{M}$ is a tight $K^{*}$ frame, since

$$
\begin{aligned}
S_{\Theta} x & =\sum_{j=1}^{M}\left\langle x, \theta_{j}\right\rangle \theta_{j} \\
& =\sum_{j=1}^{M}\left\langle x, K^{*} S_{\Phi}^{-\frac{1}{2}} P_{S_{\Phi}^{\frac{1}{2}}(R(K))} \varphi_{j}\right\rangle K^{*} S_{\Phi}^{-\frac{1}{2}} P_{S_{\Phi}^{\frac{1}{2}}(R(K))} \varphi_{j} \\
& =K^{*} S_{\Phi}^{-\frac{1}{2}} P_{S_{\Phi}^{\frac{1}{2}}(R(K))} \sum_{j=1}^{M}\left\langle x, K^{*} S_{\Phi}^{-\frac{1}{2}} P_{S_{\Phi}^{\frac{1}{2}}(R(K))} \varphi_{j}\right\rangle \varphi_{j} \\
& =K^{*} S_{\Phi}^{-\frac{1}{2}} P_{S_{\Phi}^{\frac{1}{2}}(R(K))} \sum_{j=1}^{M}\left\langle\left(S_{\Phi}^{-\frac{1}{2}}\right)^{*} K x, \varphi_{j}\right\rangle \varphi_{j} \\
& =K^{*} S_{\Phi}^{-\frac{1}{2}} P_{S_{\Phi}^{\frac{1}{2}}(R(K))} S_{\Phi}\left(S_{\Phi}^{-\frac{1}{2}}\right)^{*} K x \\
& =K^{*} S_{\Phi}^{-\frac{1}{2}} P_{S_{\Phi}^{\frac{1}{2}}(R(K))} S_{\Phi}^{\frac{1}{2}} S_{\Phi}^{\frac{1}{2}}\left(S_{\Phi}^{-\frac{1}{2}}\right)^{*} K x \\
& =K^{*} S_{\Phi}^{\frac{1}{2}}\left(S_{\Phi}^{-\frac{1}{2}}\right)^{*} K x \\
& =K^{*}\left(S_{\Phi}^{-\frac{1}{2}} S_{\Phi}^{\frac{1}{2}}\right)^{*} K x \\
& =K^{*} K x
\end{aligned}
$$

So we have $\left\langle S_{\Theta} x, x\right\rangle=\left\langle K^{*} K x, x\right\rangle=\langle K x, K x\rangle=\|K x\|^{2}$. Also, the synthesis operator associated with $\left\{\theta_{j}\right\}_{j=1}^{M}$ is $K^{*} S_{\Phi}^{-\frac{1}{2}} P_{S_{\Phi}^{\frac{1}{2}}(R(K))} T_{\Phi}^{*}$ and the synthesis operator associated with $\left\{\gamma_{j}\right\}_{j=1}^{M}$ is $\left.S_{\Phi}^{\frac{1}{2}}\left(\left.K^{\dagger}\right|_{R(K)}\right)\right)^{*} T_{\Psi}^{*}$. Since

$$
\begin{aligned}
T_{\Theta}^{*}\left(\left\{c_{j}\right\}_{j=1}^{M}\right) & =\sum_{j=1}^{M} c_{j} K^{*} S_{\Phi}^{-\frac{1}{2}} P_{S_{\Phi}^{\frac{1}{2}}(R(K))} \varphi_{j} \\
& =K^{*} S_{\Phi}^{-\frac{1}{2}} P_{S_{\Phi}^{\frac{1}{2}}(R(K))} \sum_{j=1}^{M} c_{j} \varphi_{j} \\
& =K^{*} S_{\Phi}^{-\frac{1}{2}} P_{S_{\Phi}^{\frac{1}{2}}(R(K))} T_{\Phi}^{*}
\end{aligned}
$$

We know that $\left.K^{\dagger}\right|_{R(K)}: R(K) \longrightarrow \mathscr{H}^{N}$, thus $\left(\left.K^{\dagger}\right|_{R(K)}\right)^{*}: \mathscr{H}^{N} \longrightarrow R(K)$. So

$$
\begin{aligned}
T_{\Theta}^{*} T_{\Gamma} & =K S_{\Phi}^{-\frac{1}{2}} P_{S_{\Phi}^{\frac{1}{2}}(R(K))} T_{\Phi}^{*}\left(S_{\Phi}^{\frac{1}{2}}\left(\left.K^{\dagger}\right|_{R(K)}\right)^{*} T_{\Psi}^{*}\right)^{*} \\
& =\left.K S_{\Phi}^{-\frac{1}{2}} P_{S_{\Phi}^{\frac{1}{2}}(R(K))} T_{\Phi}^{*} T_{\Psi} K^{\dagger}\right|_{R(K)} S_{\Phi}^{\frac{1}{2}}
\end{aligned}
$$




$$
\begin{aligned}
& =\left.K S_{\Phi}^{-\frac{1}{2}} P_{S_{\Phi}^{\frac{1}{2}}(R(K))} K I_{N \times N} K^{\dagger}\right|_{R(K)} S_{\Phi}^{\frac{1}{2}} \\
& =K S_{\Phi}^{-\frac{1}{2}} P_{S_{\Phi}^{\frac{1}{2}}(R(K))} S_{\Phi}^{\frac{1}{2}} \\
& =K .
\end{aligned}
$$

Therefore $\Theta=\left\{\theta_{j}\right\}_{j=1}^{M}$ and $\Gamma=\left\{\gamma_{j}\right\}_{j=1}^{M}$ are $K$-dual frame pairs. Moreover, we have $T_{\Theta}^{*} T_{\Gamma}=K=$ $T_{\Phi}^{*} T_{\Psi}$, that gives

$$
\operatorname{diag}\left(T_{\Phi}^{*} T_{\Psi}\right)=\operatorname{diag}\left(T_{\Theta}^{*} T_{\Gamma}\right)=v=\sum_{j=1}^{M}\left\langle\varphi_{j}, \psi_{j}\right\rangle=\operatorname{Tr}(K)
$$

In the following results we characterize $K$-duals of a $K$-frame.

Proposition 3.5. Let $\Phi=\left\{\varphi_{j}\right\}_{j=1}^{M}$ be a $K$-frame for $\mathscr{H}^{N}$. Then $\Psi=\left\{\psi_{j}\right\}_{j=1}^{M}$ is a $K$-dual for $\Phi$ if and only if $R\left(T_{\Phi}\right) \perp R\left(T_{\Theta}\right)$, where $T_{\Theta}$ is the analysis operator of the sequence $\Theta=\left\{\theta_{j}\right\}_{j=1}^{M}=$ $\left\{\psi_{j}-K^{*} S_{\Phi}^{-1} P_{S_{\Phi}(R(K))} \varphi_{j}\right\}_{j=1}^{M}$.

Proof. Let $\Phi=\left\{\varphi_{j}\right\}_{j=1}^{M}$ be a $K$-frame for $\mathscr{H}^{N}$ with $K$-dual $\Psi=\left\{\psi_{j}\right\}_{j=1}^{M}$, then

$$
\begin{aligned}
K x & =\sum_{j=1}^{M}\left\langle x, \psi_{j}\right\rangle \varphi_{j} \\
& =\sum_{j=1}^{M}\left\langle x, \psi_{j}-K^{*} S_{\Phi}^{-1} P_{S_{\Phi}(R(K))} \varphi_{j}+K^{*} S_{\Phi}^{-1} P_{S_{\Phi}(R(K))} \varphi_{j}\right\rangle \varphi_{j} \\
& =\sum_{j=1}^{M}\left\langle x, \theta_{j}\right\rangle \varphi_{j}+\sum_{j=1}^{M}\left\langle x, K^{*} S_{\Phi}^{-1} P_{S_{\Phi}(R(K))} \varphi_{j}\right\rangle \varphi_{j} \\
& =\sum_{j=1}^{M}\left\langle x, \theta_{j}\right\rangle \varphi_{j}+\sum_{j=1}^{M}\left\langle K x, S_{\Phi}^{-1} P_{S_{\Phi}(R(K))} \varphi_{j}\right\rangle \varphi_{j} \\
& =T_{\Phi}^{*} T_{\Theta} x+K x .
\end{aligned}
$$

Recall that two $K$-frames $\Phi=\left\{\phi_{j}\right\}_{j=1}^{M}$ and $\Psi=\left\{\psi_{j}\right\}_{j=1}^{M}$ in a Hilbert space $\mathscr{H}^{N}$ are isomorphic $K$-frames if there exists an invertible operator $U: \mathscr{H}^{N} \longrightarrow \mathscr{H}^{N}$ so that $U \phi_{j}=\psi_{j}$ for all $1 \leq j \leq M$.

Proposition 3.6. Let $K \in B\left(\mathscr{H}^{N}\right)$ and $\Phi=\left\{\varphi_{j}\right\}_{j=1}^{M}$ be a $K$-frame for $R\left(K^{*}\right)$ with the two different $K$-dual frames $\Psi=\left\{\psi_{j}\right\}_{j=1}^{M}$ and $\Gamma=\left\{\gamma_{j}\right\}_{j=1}^{M}$. Then $\Psi$ and $\Gamma$ are not isomorphic.

Proof. Assume that $\Psi=\left\{\psi_{j}\right\}_{j=1}^{M}$ and $\Gamma=\left\{\gamma_{j}\right\}_{j=1}^{M}$ are isomorphic. Hence there exists an invertible operator $U \in B\left(\mathscr{H}^{N}\right)$ satisfying $U \psi_{j}=\gamma_{j}, j=1,2, \ldots, M$. Now, for any $x \in R\left(K^{*}\right)$ we can write

$$
K U^{*} x=\sum_{j=1}^{M}\left\langle U^{*} x, \gamma_{j}\right\rangle \varphi_{j}=\sum_{j=1}^{M}\left\langle x, U \gamma_{j}\right\rangle \varphi_{j}=\sum_{j=1}^{M}\left\langle x, \psi_{j}\right\rangle \varphi_{j}=K x
$$


Therefore $\left.U\right|_{R\left(K^{*}\right)}=I d$ which is a contradiction.

Proposition 3.7. Let $K \in B\left(H^{N}\right)$. Let $\Phi=\left\{\varphi_{j}\right\}_{j=1}^{M}$ be a $K$-frame for $R\left(K^{*}\right)$, then the only $K$-dual frame of $\Phi$, which is isomorphic to $\Phi$ is $\left\{K^{*} S_{\Phi}^{-1} P_{S_{\Phi}(R(K))} \varphi_{j}\right\}_{j=1}^{M}$.

Proof. Suppose that $\Psi=\left\{\psi_{j}\right\}_{j=1}^{M}$ is a $K$-dual frame of $\Phi$ and there is an invertible operator $U$ so that $\psi_{j}=U K^{*} S_{\Phi}^{-1} P_{S_{\Phi}(R(K))} \varphi_{j}$ for all $\mathrm{i}=1,2$, M. Then, for every $x \in R\left(K^{*}\right)$ we have

$$
\begin{aligned}
K U^{*} x & =\sum_{j=1}^{M}\left\langle K U^{*} x, S_{\Phi}^{-1} P_{S_{\Phi}(R(K))} \varphi_{j}\right\rangle \varphi_{j} \\
& =\sum_{j=1}^{M}\left\langle x, U K^{*} S_{\Phi}^{-1} P_{S_{\Phi}(R(K))} \varphi_{j}\right\rangle \varphi_{j} \\
& =\sum_{j=1}^{M}\left\langle x, \psi_{j}\right\rangle \varphi_{j}=K x .
\end{aligned}
$$

Hence $\left.U\right|_{R\left(K^{*}\right)}=I_{N \times N}$. Thus

$$
\psi_{j}=U K^{*} S_{\Phi}^{-1} P_{S_{\Phi}(R(K))} \varphi_{j}=K^{*} S_{\Phi}^{-1} P_{S_{\Phi}(R(K))} \varphi_{j}
$$

Oblique dual frames were proposed by several researchers. In particular, oblique dual frames in finite dimensional Hilbert space were studied in [18]. In the last part of this section, we study this notion for $K$-frames.

Definition 3.8. Let $\mathscr{U}$ and $\mathscr{W}$ be two subspaces of $\mathscr{H}^{N}$ and suppose that $\Phi=\left\{\varphi_{j}\right\}_{j=1}^{M}$ and $\Psi=\left\{\psi_{j}\right\}_{j=1}^{M}$ are in $\mathscr{H}^{N}$ and $\mathbb{W}=\operatorname{span}\left\{\varphi_{j}: j=1,2, \ldots, M\right\}, \mathscr{U}=\operatorname{span}\left\{\psi_{j}: j=1,2, \ldots, M\right\}$. The sequence $\Psi=\left\{\psi_{j}\right\}_{j=1}^{M}$ is an oblique $K$-dual frame of the $K$-frame $\Phi=\left\{\varphi_{j}\right\}_{j=1}^{M}$ on $\mathbb{W}$ if

$$
K x=\sum_{j=1}^{M}\left\langle x, \psi_{j}\right\rangle \varphi_{j}, \quad(x \in \mathbb{W}) .
$$

Note that (3.5) implies that $R(K \mid \mathbb{W}) \subseteq \mathbb{W}$.

In the following two propositions a characterization of the oblique $K$-dual frames pair that are obtained by adding a pair of vector sequences to a given Bessel sequence is given. Also, characterize the uniqueness of the oblique $K$-dual frame pair.

Proposition 3.9. Suppose that $\mathbb{W}$ is a subspace of $\mathscr{H}^{N}$ and sequences $\Phi=\left\{\varphi_{j}\right\}_{j=1}^{M}, \Psi=\left\{\psi_{j}\right\}_{j=1}^{L}$ and $\Gamma=\left\{\gamma_{j}\right\}_{j=1}^{L}$ in $\mathscr{H}^{N}$ satisfy that $\operatorname{span}(\Phi \cup \Gamma)=\mathscr{W}$. Then the following statements are equivalent:

(i) $\Phi \cup \Psi$ is an oblique $K$-dual frame of $\Phi \cup \Gamma$ on $\mathbb{W}$.

(ii) For any $x \in \mathbb{W},\left(K-S_{\Phi}\right) x=\sum_{j=1}^{L}\left\langle x, \psi_{j}\right\rangle \gamma_{j}$. 
Proof. (i) and (ii) are equivalent, since $\Phi \cup \Psi$ is an oblique $K$-dual frame of $\Phi \cup \Gamma$ on $\mathbb{W}$, if and only for all $x \in \mathbb{W}$

$$
\begin{aligned}
K x & =\sum_{j=1}^{M}\left\langle x, \varphi_{j}\right\rangle \varphi_{j}+\sum_{j=1}^{L}\left\langle x, \psi_{j}\right\rangle \gamma_{j} \\
& =S_{\Phi} x+\sum_{j=1}^{L}\left\langle x, \psi_{j}\right\rangle \gamma_{j},
\end{aligned}
$$

so

$$
\left(K-S_{\Phi}\right) x=\sum_{j=1}^{L}\left\langle x, \psi_{j}\right\rangle \gamma_{j}
$$

Proposition 3.10. If $\Psi=\left\{\psi_{j}\right\}_{j=1}^{M}$ is an oblique $K$-dual frame of $\Phi=\left\{\varphi_{j}\right\}_{j=1}^{M}$ on $\mathbb{W}$ and $\Phi$ is $K$-minimal, then the oblique $K$-dual frame of $\Phi$ on $\mathbb{W}$ is unique in the sense that if $\Gamma=\left\{\gamma_{j}\right\}_{j=1}^{M}$ is another oblique $K$-dual frame of $\Phi$, then $\psi_{j}=\gamma_{j}, j=1, \ldots, M$, where $\Psi, \Gamma$ are restricted in $W$.

Proof. Due to the fact that $\Psi=\left\{\psi_{j}\right\}_{j=1}^{M}$ and $\Gamma=\left\{\gamma_{j}\right\}_{j=1}^{M}$ are oblique $K$-dual frames of $\Phi$ on $\mathbb{W}$, then we can write

$$
K x=\sum_{j=1}^{M}\left\langle x, \psi_{j}\right\rangle \varphi_{j}=\sum_{j=1}^{M}\left\langle x, \gamma_{j}\right\rangle \varphi_{j},(x \in \mathbb{W}) .
$$

Hence $\sum_{j=1}^{M}\left\langle x, \psi_{j}-\gamma_{j}\right\rangle \varphi_{j}=0, x \in \mathbb{W}$. Now by $K$-minimality of $\Phi$, we have

$$
\left\langle x, \psi_{j}-\gamma_{j}\right\rangle=0, j=1,2, \ldots, M, x \in \mathbb{W},
$$

and therefore $\psi_{j}=\gamma_{j}, j=1, \ldots, M$.

Here, we state that if $\Phi$ is a $K$-frame for $R(K)$, then we can make an oblique $K$-dual frame of algebraic multiplicity of $\left\{\varphi_{j}\right\}_{j=1}^{M} \cup\left\{e_{j}\right\}_{j \neq j_{0}}$ where $\left\{e_{j}\right\}_{j=1}^{d}$ is an orthonormal eigenbasis of the frame operator $S_{\Phi}$ with associated eigenvalues $\left\{\lambda_{j}\right\}_{j=1}^{d}$.

Theorem 3.11. Let $K \in B\left(\mathscr{H}^{N}\right)$ and $\Phi=\left\{\varphi_{j}\right\}_{j=1}^{M}$ be a $K$-frame for $\mathbb{W}=R(K)$ with dimW $=d$. Let $\left\{e_{j}\right\}_{j=1}^{d}$ be an orthonormal eigenbasis of the frame operator $S_{\Phi}$ with associated eigenvalues $\left\{\lambda_{j}\right\}_{j=1}^{d}$. Then for any eigenvalue $0 \neq \lambda_{j_{0}}$, the sequence $\left\{\frac{1}{\sqrt{\lambda_{j_{0}}}} K^{*} \varphi_{j}\right\}_{j=1}^{M} \cup\left\{\frac{\left(\lambda_{j_{0}}-\lambda_{j}\right)^{\frac{1}{3}}}{\sqrt{\lambda_{j_{0}}}} K^{*} e_{j}+\right.$ $\left.K^{*} \gamma_{j}\right\}_{j \neq j_{0}}$, is an oblique $K$-dual frame of $\left\{\frac{1}{\sqrt{\lambda_{j_{0}}}} \varphi_{j}\right\}_{j=1}^{M} \cup\left\{\frac{\left(\lambda_{j_{0}}-\lambda_{j}\right)^{\frac{2}{3}}}{\sqrt{\lambda_{j_{0}}}} e_{j}\right\}_{j \neq j_{0}}$ on $\mathbb{W}$, where $\left\{\gamma_{j}\right\}_{j_{0} \neq j=1}^{d}$ $\subset \mathscr{H}^{N}$ satisfies

$$
\sum_{j_{0} \neq j=1}^{d}\left\langle x, K^{*} \gamma_{j}\right\rangle e_{j}=0,(x \in \mathbb{W}) .
$$

Proof. Let $\Phi$ be a $K$-frame for $\mathbb{W}=R(K)$ with the frame operator $S_{\Phi}$. Also $\left\{e_{j}\right\}_{j=1}^{d}$ is an orthonormal eigenbasis, so for any $x \in \mathbb{W}$ we can write $K x=\sum_{j=1}^{d}\left\langle K x, e_{j}\right\rangle e_{j}$. Thus

$$
S_{\Phi} K x=\sum_{j=1}^{M}\left\langle K x, \varphi_{j}\right\rangle \varphi_{j}=S_{\Phi} \sum_{j=1}^{d}\left\langle K x, e_{j}\right\rangle e_{j}
$$




$$
\begin{aligned}
& =\sum_{j=1}^{d}\left\langle K x, e_{j}\right\rangle S_{\Phi} e_{j}=\sum_{j=1}^{d}\left\langle K x, e_{j}\right\rangle \lambda_{j} e_{j} \\
& =\sum_{j=1}^{d} \lambda_{j}\left\langle K x, e_{j}\right\rangle e_{j} .
\end{aligned}
$$

Now for all $x \in \mathbb{W}$ we have

$$
\begin{aligned}
& K x=\frac{1}{\lambda_{j_{0}}} \sum_{j=1}^{d} \lambda_{j_{0}}\left\langle K x, e_{j}\right\rangle e_{j} \\
& =\frac{1}{\lambda_{j_{0}}}\left(\lambda_{j_{0}}\left\langle K x, e_{j_{0}}\right\rangle e_{j_{0}}+\sum_{j \neq j_{0}} \lambda_{j_{0}}\left\langle K x, e_{j}\right\rangle e_{j}\right) \\
& =\frac{1}{\lambda_{j_{0}}}\left(\lambda_{j_{0}}\left\langle K x, e_{j_{0}}\right\rangle e_{j_{0}}+\sum_{j \neq j_{0}} \lambda_{j}\left\langle K x, e_{j}\right\rangle e_{j}-\sum_{j \neq j_{0}} \lambda_{j}\left\langle K x, e_{j}\right\rangle e_{j}\right. \\
& \left.+\sum_{j \neq j_{0}} \lambda_{j_{0}}\left\langle K x, e_{j}\right\rangle e_{j}\right) \\
& =\frac{1}{\lambda_{j_{0}}}\left(\lambda_{j_{0}}\left\langle K x, e_{j_{0}}\right\rangle e_{j_{0}}+\sum_{j \neq j_{0}} \lambda_{j}\left\langle K x, e_{j}\right\rangle e_{j}\right. \\
& \left.+\sum_{j \neq j_{0}}\left(\lambda_{j_{0}}-\lambda_{j}\right)\left\langle K x, e_{j}\right\rangle e_{j}\right) \\
& =\frac{1}{\lambda_{j_{0}}}\left(\sum_{j=1}^{d} \lambda_{j}\left\langle K x, e_{j}\right\rangle e_{j}+\sum_{j \neq j_{0}}\left(\lambda_{j_{0}}-\lambda_{j}\right)\left\langle K x, e_{j}\right\rangle e_{j}\right) \\
& =\frac{1}{\lambda_{j_{0}}} \sum_{j=1}^{M}\left\langle K x, \varphi_{j}\right\rangle \varphi_{j}+\sum_{j \neq j_{0}}\left(\frac{\lambda_{j_{0}}-\lambda_{j}}{\lambda_{j_{0}}}\right)\left\langle K x, e_{j}\right\rangle e_{j} \\
& =\frac{1}{\lambda_{j_{0}}} \sum_{j=1}^{M}\left\langle K x, \varphi_{j}\right\rangle \varphi_{j}+\sum_{j \neq j_{0}} \frac{\left(\lambda_{j_{0}}-\lambda_{j}\right)^{\frac{1}{3}}}{\sqrt{\lambda_{j_{0}}}} \frac{\left(\lambda_{j_{0}}-\lambda_{j}\right)^{\frac{2}{3}}}{\sqrt{\lambda_{j_{0}}}}\left\langle K x, e_{j}+\gamma_{j}\right\rangle e_{j} \\
& =\frac{1}{\lambda_{j_{0}}} \sum_{j=1}^{M}\left\langle K x, \varphi_{j}\right\rangle \varphi_{j}+\sum_{j \neq j_{0}}\left\langle K x, \frac{\left(\lambda_{j_{0}}-\lambda_{j}\right)^{\frac{1}{3}}}{\sqrt{\lambda_{j_{0}}}} e_{j}+\gamma_{j}\right\rangle \frac{\left(\lambda_{j_{0}}-\lambda_{j}\right)^{\frac{2}{3}}}{\sqrt{\lambda_{j_{0}}}} e_{j} \\
& =\sum_{j=1}^{M}\left\langle K x, \frac{1}{\sqrt{\lambda_{j_{0}}}} \varphi_{j}\right\rangle \frac{1}{\sqrt{\lambda_{j_{0}}}} \varphi_{j} \\
& +\sum_{j \neq j_{0}}\left\langle K x, \frac{\left(\lambda_{j_{0}}-\lambda_{j}\right)^{\frac{1}{3}}}{\sqrt{\lambda_{j_{0}}}} e_{j}+\gamma_{j}\right\rangle \frac{\left(\lambda_{j_{0}}-\lambda_{j}\right)^{\frac{2}{3}}}{\sqrt{\lambda_{j_{0}}}} e_{j} \\
& =\sum_{j=1}^{M}\left\langle x, \frac{1}{\sqrt{\lambda_{j_{0}}}} K^{*} \varphi_{j}\right\rangle \frac{1}{\sqrt{\lambda_{j_{0}}}} \varphi_{j} \\
& +\sum_{j \neq j_{0}}\left\langle x, \frac{\left(\lambda_{j_{0}}-\lambda_{j}\right)^{\frac{1}{3}}}{\sqrt{\lambda_{j_{0}}}} K^{*} e_{j}+K^{*} \gamma_{j}\right\rangle \frac{\left(\lambda_{j_{0}}-\lambda_{j}\right)^{\frac{2}{3}}}{\sqrt{\lambda_{j_{0}}}} e_{j},
\end{aligned}
$$

which complete the proof. 


\section{References}

[1] F. Arabyani and A. A. Arefijamaal, Some constructions of K-frames and their duals, To appear in Rocky Mountain J. Math.https://projecteuclid.org/euclid.rmjm/1455560377.

[2] J. J. Benedetto and M. Fickus, Finite normalized tight frames, Adv. Comput. Math., 18 (2003), 357-385.

[3] H. Bolcskei, F. Hlawatsch and H. G. Feichtinger, Frame-theoretic analysis of over-sampled filter banks, IEEE Trans. Signal Process. 46 (1998), 3256-3268.

[4] P. Casazza, G. Kutyniok and F. Philipp, Finite Frames: Theory and Applications. Birkhäuser, Berlin, 2013.

[5] O. Christensen, A. M. Powell and X. C. Xiao, A note on finite dual frame pairs, Proc. Amer. Math. Soc., 140 (11) (2012), 3921-3930.

[6] O. Christensen, Frames and Bases: An Introductory Course, Birkhäuser, Boston 2008.

[7] I. Daubechies, A. Grossmann and Y. Meyer, Painless non-orthogonal expansions, J. Math. Physics, 27 (1986), 1271-1283.

[8] R. J. Duffin and A. C. Schaeffer, A class of nonharmonic Fourier series, Trans. Amer. Math. Soc., 72 (1952), 341-366.

[9] Y. C. Eldar and G. D. Forney Jr., Optimal tight frames and quantum measurement. IEEE Trans. Inform. Theory, 48 (3) (2002), 599-610.

[10] P. J. S. G. Ferreira, Mathematics for multimedia signal processing II: Discrete finite frames and signal reconstruction, In: Byrnes, J.S. (ed.) Signal processing for multimedia, IOS Press, Amsterdam (1999), 35-54.

[11] L. Găvruţa, Frames for operators, Appl. Comput. Harmon. Anal., 32 (2012), 139-144.

[12] L. Găvruţa, Atomic decompositions for operators in reproducing kernel Hilbert spaces, Math. Rep., 17 (3) (2015), 303-314.

[13] K. Gröchenig and C. Heil, Modulation spaces and pseudodifferential operators. Integral Equ. Oper. Theory 34 (4) (1999), 439-457.

[14] D. Han and D. Larson, Frames, Bases and group representations, Mem. Amer. Math. Soc., 147 (697) 2000.

[15] R. W. Heath Jr. and A. Paulraj, Linear dispersion codes for MIMO systems based on frame theory. IEEE Trans. Signal Process., 50 (10) (2002), 2429-2441.

[16] Zh. Q. Xiang and Y. M. Li, Frame sequences and dual frames for operators, ScienceAsia, 42 (2016), $222-230$.

[17] X. Xiao, Y. Zhu and L. Găvruţa, Some properties of K-frames in Hilbert spaces, Results. Math., 63 (3-4) (2013), 1243-1255.

[18] X. C. Xiao, Y. C. Zhu and X. M. Zeng, Oblique dual frames in finite-dimensional Hilbert spaces, Int. J. Wavelets Multiresolut. Inf. Process, 11 (2) (2013), 1350011-1-1350011-14.

Department of Pure Mathematics, Ferdowsi University of Mashhad, Mashhad, P.O. Box 1159-91775, Iran.

E-mail: va_mo584@stu.um.ac.ir

Department of Pure Mathematics, Ferdowsi University of Mashhad, Mashhad, P.O. Box 1159-91775, Iran.

E-mail: janfada@um.ac.ir

Department of Pure Mathematics, Ferdowsi University of Mashhad, Mashhad, P.O. Box 1159-91775, Iran.

E-mail: kamyabi@um.ac.ir 